\title{
Mechanistic Insights into the Superexchange-Interaction-Driven Negative Thermal Expansion in $\mathrm{CuO}$
}

\author{
Yuanpeng Zhang ${ }^{\mathrm{a}, \mathrm{b}}$, Marshall McDonnell ${ }^{\mathrm{a}}$, Stuart A. Calder ${ }^{\mathrm{a}}$, and Matthew G. Tucker ${ }^{\mathrm{a}}$
}

${ }^{a}$ Neutron Scattering Division, Oak Ridge National Laboratory (ORNL), Oak Ridge, Tennessee 37831, United States, zhang.yuanpeng@nist.gov

${ }^{b}$ Materials Measurement Science Division, National Institute of Standards and Technology (NIST), 100 Bureau Drive, Gaithersburg, Maryland 20899, United States, zhang.yuanpeng@nist.gov

The negative thermal expansion (NTE) in $\mathrm{CuO}$ is explained via electron-transfer-driven superexchange interaction. The elusive connection between the spin-lattice coupling and NTE of $\mathrm{CuO}$ is investigated by neutron scattering and principal strain axes analysis. The density functional theory calculations show as the temperature decreases, the continuously increasing electron transfer accounts for enhancing the superexchange interaction along [101] , the principal NTE direction. It is further rationalized that only when the interaction along $[10 \overline{1}]$ is preferably enhanced to a certain level compared to the other competing antiferromagnetic exchange pathways can the corresponding NTE occur. Outcomes from this work have implications for controlling the thermal expansion through superexchange interaction, via, for example, optical manipulation, electron or hole doping, etc.

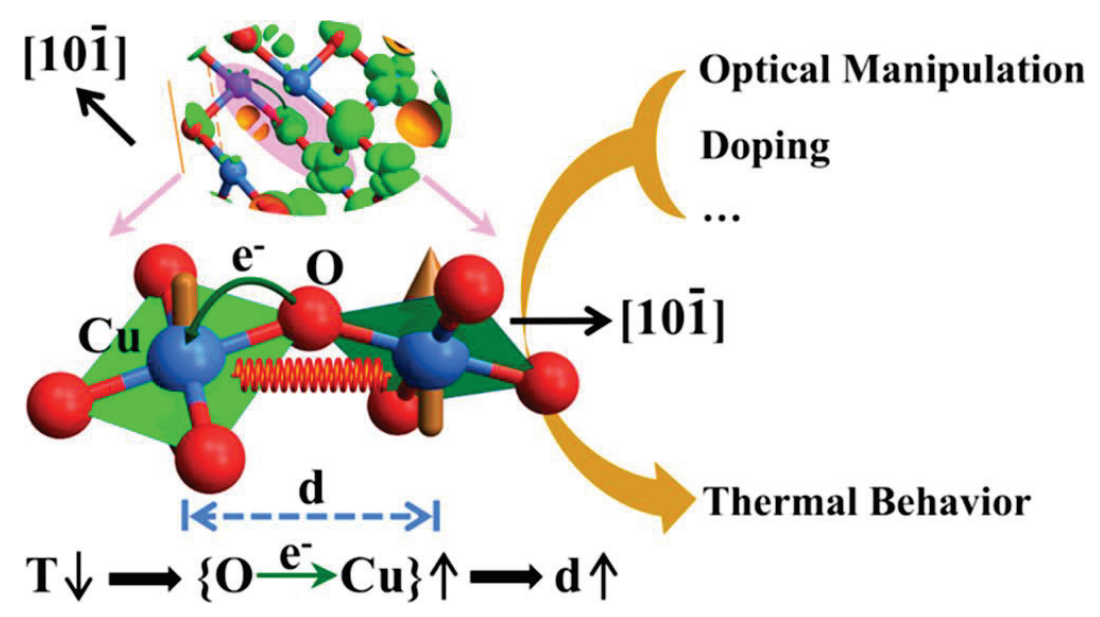

Fig. 1. Illustration for superexchange-interaction-driven negative thermal expansion in $\mathrm{CuO}$.

References

[1] Zhang, Y. P., et al. (2019), J. Am. Chem. Soc., Article ASAP, XXXX, XXX, XXX-XXX 